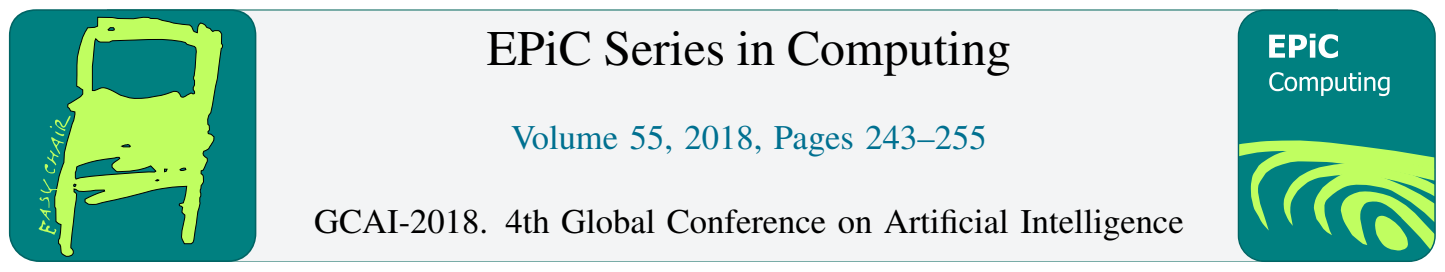

\title{
Replaceability for Constraint Satisfaction Problems: Algorithms, Inferences, and Complexity Patterns
}

\author{
Richard J. Wallace \\ Insight Centre for Data Analytics \\ Department of Computer Science, University College Cork, Cork, Ireland \\ richard.wallacedinsight-centre.org
}

\begin{abstract}
Replaceability is a form of generalized substitutability whose features make it potentially of great importance for problem simplification. It differs from simple substitutability in that it only requires that substitutable values exist for every solution containing a given value without requiring that the former always be the same. This is the most general form of substitutability that allows inferences from local to global versions of this property. Building on earlier work, this study first establishes that algorithms for localized replaceability (consistent neighbourhood replaceability or CNR algorithms) based on all-solutions neighbourhood search outperform other replaceability algorithms by several orders of magnitude. It also examines the relative effectiveness of different forms of depth-first CNR algorithms. Secondly, it demonstrates an apparent complexity ridge, which does not occur at the same place in the problem space as the complexity areas for consistency or full search algorithms. Thirdly, it continues the study of methods for inferring replaceability in structured problems in order to improve efficiency. Here, it is shown that some strategies for inferring replaceable values can be extended to disjunctive constraints in scheduling problems.
\end{abstract}

\section{Introduction}

Among the many methods devised for simplifying constraint satisfaction problems, those based on substitutability form a significant class. In contrast to methods that discover and remove inconsistent values, i.e. values that cannot appear in any solution, substitutability methods remove values that may be perfectly viable. However, in these cases other viable values can be substituted for the value removed without affecting the validity of the solution. Hence, removal depends on the fact that these values are redundant with respect to problem satisfiability.

As with consistency methods, there is now a body of work concerned with specifying various forms of substitutability as well as related properties such as interchangeability or mutual substitutability. A useful summary of this work is found in [10]. One of the most interesting developments in this area is the demonstration that generalized forms of substitutability can be defined that effectively loosen the criteria for value removal without affecting the guarantee that, given a solution with those values, other solutions can still be found $[8,1,6]$.

Recently it was shown that some important substitutability properties can be arranged into a wellformed hierarchy, which was called the substitutability hierarchy. It was also possible to show that 
within that hierarchy a particular form of generalized substitutability has certain maximal features that make it of great potential interest. Bordeaux and co-workers called this property "removability". However, this term can lead to confusion with a still more general property called "dispensability" [4], so it seems best to use a different and perhaps more apposite term. For this purpose, we have chosen the term "replaceability", which seems to have exactly the right connotations.

Perhaps the most important feature of replaceability is that it is the most general form of substitutability that supports local to general reasoning. Unfortunately, as shown earlier and discussed below, this does not give us a form of the property that is tractable, in contrast to simple substitutability (or local consistency). Nonetheless, earlier work has shown that algorithms for local forms of replaceability can be devised that are feasible for some problems [6].

Building from the results reported in [6], the present work extends the analysis of this property. In the first place, we consider other algorithms that have been proposed in recent years to determine whether they are reasonable alternatives to the algorithms we devised. Then we examine a potentially significant critical complexity effect found with replaceability algorithms. Although it is based on a dynamics that is similar to the well-known phase transition, involving a tradeoff between number of possible tuples and a decreasing likelihood that any one of them will be viable, it does not involve a phase transition. Finally, we extend earlier work on inferring replaceability to the important class of scheduling problems with disjunctive constraints.

In the past, there have been a few efforts in this direction. The earliest is the work by Jeavons et al., who defined what they called "generalized substitutability" [8]. Later Bordeaux et al. defined a similar but simpler concept that they called "removability" [1], which is identical to replaceability as we define it here. Then Freuder showed that replaceability could support certain forms of local to global reasoning [4]. Subsequently, Likitvivatanavong and Yap discussed the same idea and proposed a new algorithm for removing all replaceable values [11]. As noted above, Freuder and Wallace recently delineated a "substitutability hierarchy" and showed that replaceability is the most general property in this hierarchy that can support local to global reasoning. These authors also proposed two new algorithms for local replaceability based on depth-first search [6].

\section{Background and Basic Concepts}

A constraint satisfaction problem (CSP) involves choosing values for a set of variables, $X$, which satisfy a set of constraints, $C$, each of which specifies permissible combinations of values for a subset of the variables. Each value selected for variable $X_{i}$ must come from a domain of values $D_{i}$ associated with that variable. A choice of values for a subset, $S$, of the variables is an instantiation of $S$. If the instantiation is consistent with constraints based on variables in $S$, then we say that it satisfies those constraints. An instantiation of all the variables, $X$, which satisfies all the constraints in the problem is a solution.

CSPs can be simplified by removing values that are "inconsistent", i.e. that cannot appear together with any value in any solution. In addition, simplification can be effected if whenever a value appears in a solution, another value can be found to substitute for it without making the solution invalid.

The basic property of substitutability in CSPs was first characterised in [3]. Here, we will use an equivalent definition that corresponds to later definitions in which the focus is on the value discarded.

Definition 1. Given a value $v$ in domain $D_{i}$ of variable $X_{i}$, if for any solution in which $v$ appears, we can substitute $u$ and still have a solution, then $v$ has the property of substitutability and value $u$ is substitutable for $v$.

The same work introduced a local form of substitutability, called neighbourhood substitutability. This depends on the idea of the neighbourhood of a variable $X_{i}$, which is the set of variables adjacent 
to $X_{i}$ in the constraint graph. These are the variables that share a constraint with $X_{i}$.

Definition 2. (From [3]) For two values $u$ and $v$ belonging to the domain of variable $X_{i}, u$ is neighbourhood substitutable for $v$ iff for every constraint on $X_{i}$, if $v$ satisfies the constraint then $u$ also satisfies it.

This latter property had two important features: (i) neighbourhood substitutability is sufficient (but not necessary) for full substitutability, (ii) neighbourhood substitutability can be determined in polynomial time.

As already noted, an important generalization of substitutability holds when for a given value $v$ we can substitute some value, not necessarily unique, in any solution in which $v$ appears. This property we call replaceability. More formally,

Definition 3. (From [1]) An value $v$ is replaceable if for any solution involving $v$, there is some other instantiation that can be substituted for $v$ and still have a solution.

In other words, a value is replaceable if some value can be found to substitute for it in any solution, and it is also substitutable if a single other value can be substituted for it in every solution. Thus, substitutability implies replaceability, while replaceability does not imply substitutability. In other words, replaceability is a more general property since any values that can be removed on the basis of substitutability are also replaceable, but not vice versa.

While weaker and more general forms of substitutability can be defined, including minimal substitutability [6], these properties lack an important feature of the stronger properties. This is that up to and including replaceability, the property in question is defined with respect to all solutions in which instantiation $v$ appears, i.e. all solutions supported by a replaceable value $v$ are still supported. In particular, in [6] it was shown that:

Proposition 1. Replaceability is the most general form of substitutability for which all solutions consistent with a discarded value are consistent with some remaining value.

It can therefore be said that replaceability is a "maximal property" with respect to this feature.

In this paper we will not consider generalizations of these properties that involve $k$-tuples of values with $k>1$ and which can involve sets of values or tuples to be replaced. This is because they entail complexities in computation and representation that will make it difficult to use them in practice. In particular, as shown in [6], substitutability in terms of $k$-tuples over a variable set $R$ does not imply that individual values in the set are substitutable. In other words, the property of substitutability when applied to a $k$-tuple is not decomposable.

Removing instantiations based on these properties, either in preprocessing or dynamically during search, can reduce search effort. In general, however, determining various forms of substitutability can be as intractable as solving the original problem. However just as with substitutability, for replaceability we can define local forms that imply full replaceability (see [6] for proofs). Here, we will focus on the simplest and most useful form that we call neighbourhood replaceability.

Definition 4. An instantiation of a single variable $X_{i}$ is neighbourhood replaceable if it is replaceable with respect to the subgraph formed by $X_{i}$ and all variables that share a constraint with $X_{i}$.

Unfortunately, in contrast to substitutability, the complexity of even the local form of replaceability is non-polynomial. Nonetheless, there are cases where such computations are feasible. So we turn next to a consideration of algorithms for this task.

\section{Neighbourhood Replaceability Algorithms}

There seem to be two general approaches that one can take for computing replaceability and removing all neighbourhood replaceable values. In the first, replaceability is computed locally in a manner 


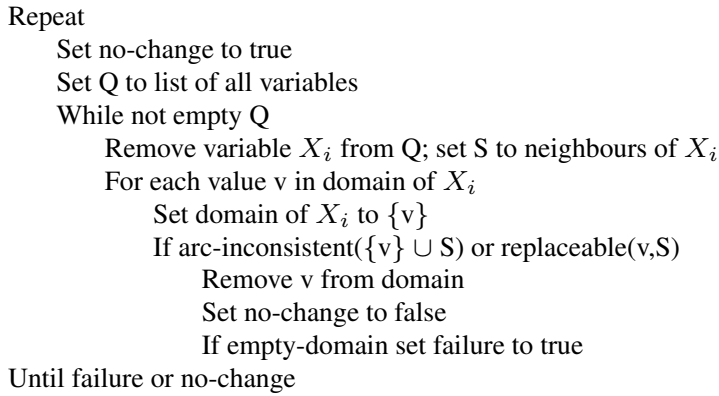

Figure 1: CNR-1 algorithm for neighbourhood replaceability.

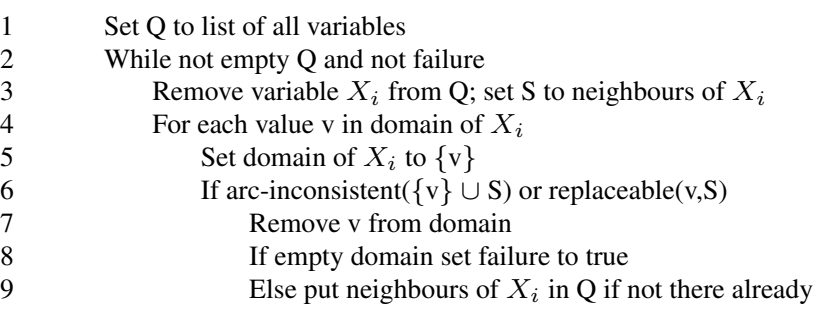

Figure 2: CNRQ algorithm for neighbourhood replaceability.

analogous to the local computation of neighbourhood inverse consistency [5]. The second approach depends heavily on inferences about the number of neighbourhood tuples associated with a given value and the number available to replace it. This is exemplified by the algorithm described by Likitvivatanavong and Yap [11].

In this section we briefly descibe the two neighbourhood search algorithms that were introduced in [6]. (There one can find soundness proofs for these algorithms.) These algorithms are designated as "consistent neighbourhood replaceability" (CNR) algorithms because they remove all values that are locally replaceable including arc-inconsistent values. In this form neighbourhood replaceability subsumes (and therefore dominates with respect to value removal) neighbourhood consistency algorithms such as neighbourhood inverse consistency (NIC) and neighbourhood singleton arc consistency (NSAC). Briefly, NIC establishes that a value $v$ of variable $X_{i}$ is consistency with at least one tuple based on $X_{i}$ and its neighbours, while NSAC determines whether the neighbourhood subgraph can be made arc consistent when $v$ is the only member of the domain of $X_{i}$. (See $[5,13]$ for more detailed discussion.) In addition, we describe a slightly altered version of the Likitvivatanavong-Yap algorithm. (Another algorithm for generalized substitutability was proposed by [8], but since it is not oriented toward finding single replaceable values, and it seems fairly inefficient, it is not described here.)

The first CNR algorithm, CNR-1, uses an AC-1 style procedure in which all values in the problem are repeatedly tested for replaceability until no value is discarded. In the implementation of this algorithm, the replaceable procedure uses a MAC-style search (MAC=maintained arc consistency) to find all solutions for the subproblem, and for each solution it seeks a value from the current domain that can replace value $v$. Pseudocode for this algorithm is shown in Figure 1. The second algorithm, CNRQ, uses a queue updating mechanism in place of the repeat loop used by CNR-1. Pseudocode for this algorithm is shown in Figure 2.

Since the algorithm described in [11] processes only a single value, the CNRQ top-level queue 


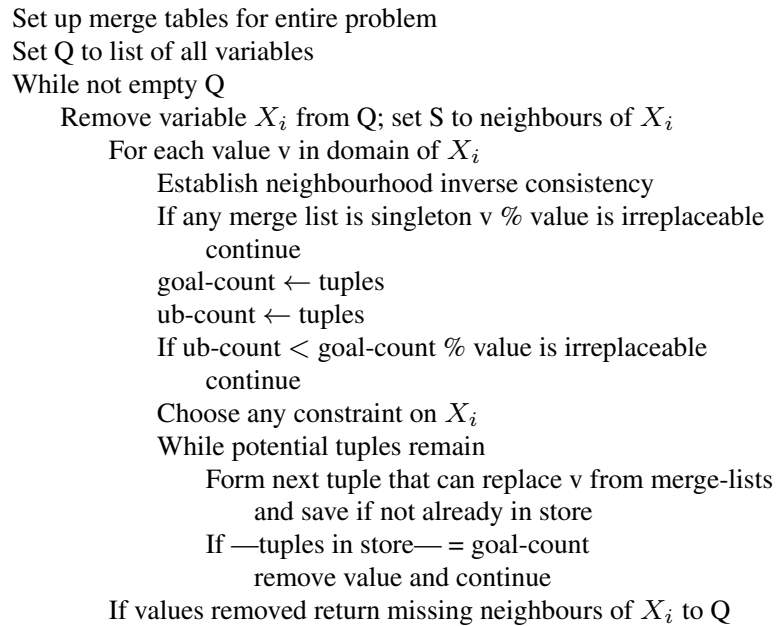

Figure 3: Likitvivatanavong-Yap (LY) algorithm for neighbourhood replaceability embedded within a top-level AC-3 style queue.

was used for handling the entire problem. In addition NIC was substituted for the maxRPC algorithm in the original description; this made the present algorithm more comparable to the other algorithms. Pseudocode for this algorithm is shown in Figure 3.

The algorithm begins with some initialization steps. The most elaborate is the setting up of merge tables for each variable in the problem. Here, we will consider only problems with binary constraints. ${ }^{1}$ In this case, the tables for each variable $X_{i}$ contain the values in the domain of $X_{i}$ that are supported by each value in the domain of each adjacent variable. Suppose, for example, that $X_{i}$ has only one neighbour $X_{j}$, that each variable has three values, and the constraint is an inequality constraint. Then the merge table would have the form,

where the values in brackets are those in the domain of $X_{i}$ that go with the value in the domain of $X_{j}$, which is indicated on the right. In this paper the values in brackets are called merge sets. (In practice, the latter value can be represented implicitly by an array index.)

In the main procedure, at each step a variable is taken off the queue and all values in its domain are checked for replaceability. Following removal of neighbourhood-inconsistent values (line 6), this algorithm carries out a series of steps, each of which determines if the value being tested is irreplaceable or replaceable. In the first test the merge sets are checked to determine if the present value is contained in a singleton; in this case, the value is irreplaceable. Prior to the next two tests, two values are computed: the number of neighbourhood tuples associated with the value being tested (goal-count) and the number of neighbourhood tuples associated with alternative values (ub-count). (In the merge table above for value 1 the goal-count is 2 and the ub-count is also 2.) Then, the next test checks whether the ub-count is less than the goal-count; if it is, then the value is irreplaceable. Finally, viable tuples are collected for

${ }^{1}$ All of these algorithms can be generalized to problems with constraints of any arity. 


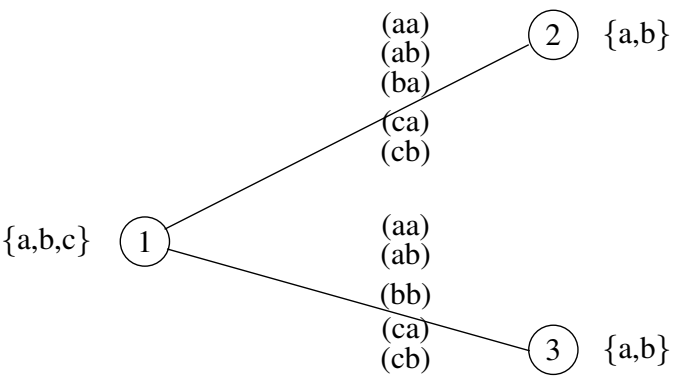

Figure 4: Example showing that more than one fixpoint is possible when all replaceable values are removed.(Domains are shown in brackets, satisfying constraint tuples are in parentheses.)

the values that could substitute for the value being tested; if ever their number equals the goal-count, then the value being tested is replaceable.

The final test is the most elaborate since it must calculate a union of tuples. In the present work two methods were tried. The first uses an array with dimensions Number-of-Adjacent-Variables X DomainSize X Number-of-Tuples. Array entries are t or nil depending on whether or not the $k$ th value in tuple $m$ has value $a$. This allows fast checking, but can require $O$ (num-neighbourhood-tuples) tuple checks. The second method uses a trie structure in which if the $k$ th value in tuple $m$ has value $a$, then at that level the (domain size) array will point to another array representing the next value in the tuple (or if $a$ is the last value, the array entry will have the value $\mathrm{t}$ ). This allows for fast checking, although there is a proliferation of small arrays each time a value is checked for replaceability. Since the second method was found to improve performance by at least an order of magnitude, this was the version used in experimental tests.

Although replaceability algorithms terminate with a set of irreplaceable values, these sets can vary depending on the order of testing. In other words, replaceability algorithms do not have unique fixpoints. This can be shown by example, as in Figure 4. In this problem, if we begin with variable 1 we can either replace value $a$ with $\{b, c\}$ or vice versa. This will then yield different fixpoints.

\section{Notes on Experimental Methods}

As stated in our earlier work, higher-order properties such as replaceability will become more useful as the field moves away from one-shot problem solving to more long-term venues involving problem compilation and/or repeated solving in evolving situations. However, we can still learn something about the effects of establishing replaceability, as well as the costs in experiments involving preprocessing and then search on individual problems.

The present tests were carried out using three types of problems:

1. Heterogeneous random problems with "geometric" constraint graphs in which the probability of support was varied ${ }^{2}$,

2. Random distance problems, i.e. problems with constraints of the form $\left|X_{i}-X_{j}\right|>k$,

\footnotetext{
${ }^{2}$ As noted earlier, homogeneous random problems have few replaceable values except in domains of variables of very low degree.
} 
3. Random relop problems, i.e. problems with (binary) constraints based on relational operators, e.g. $X_{i}>X_{j}$. Problems included not-equals constraints to ensure intractability.

Geometric problems used in the main experiments had 120 or 300 variables, both with domain size 20. Constraint graphs were constructed using the procedure described in [9], which gives the graphs a 'clumpy' character. For 120 variables the (Euclidean) distance parameter was 0.17 and problems were filtered to allow only those with 540 constraints \pm 3 . For 300 variables the distance parameter was 0.1 and the number of constraints was $1320 \pm 5$. There were two levels of support: for $70 \%$ of the values tightness (obverse of support) was 0.2 ; for $30 \%$ it was 0.8 . Support for different adjacent variables was computed independently of other constraints.

Distance problems had 50 variables, domain size 10, constraint graph density 0.10 , and fixed $k=3$. Relop problems used in the main experiments had 40 variables, domain size 10, and constraint graph density $=0.60$. Problems used in Section 7 had 30 variables, domain size 8 and varying constraint graph densities. All relop problems had $>$ and $<>$ constraints in equal proportions.

Samples of 50 problems were used except for 120 -variable geometric problems where the sample size was 100 . The parameter values used allowed samples of satisfiable and unsatisfiable problems to be generated in each case.

In these experiments search was done with MAC-3. For most problem sets the domain/forward degree variable ordering heuristic was used, while for 300-variable geometric problems the domain/ weighted degree heuristic of [2] was used.

In most tests, in addition to CNR algorithms, three other kinds of preprocessing were also performed: simple arc consistency, neighbourhood singleton arc consistency (NSAC), and neighbourhood inverse consistency (NIC). The comparisons of interest were runtimes and number of values removed.

\section{Comparing the Three CNR Algorithms}

The first set of tests was run to compare the all-solutions search algorithms with the LikitvivatanavongYap algorithm. This is important because of the very different strategies used in the two cases.

For reasons that will become evident, these experiments were run with very small problems. Random geometric problems were used with 20 variables, domain size ten, and a distance factor of 0.200 . Two levels of support were used: $60 \%$ of the values had a support $=0.8$, and the remaining $40 \%$ had a support of 0.2. Problems were filtered during generation so that, (i) all problems had $36 \pm 2$ constraints, (ii) all problems had solutions. Fifty problems were tested.

CNR algorithms removed an average of 122 values our of 200, while AC removed 16 and both NSAC and NIC removed 34. Of much greater consequence are the runtimes. CNRQ required an average of 0.12 seconds per problem; CNR- 1 was slightly slower at 0.16 seconds. (AC required $<0.01$ seconds, NSAC 0.03, NIC 0.06.) This was markedly different from CNR-LY, which required 30,382 seconds on average, so that the run for this algorithm could barely be finished.

Further insight into the differences in runtime is obtained by considering individual runtimes, which are summarized in the frequency histogram shown in Figure 5. To accomodate the large variation in a single figure, runtimes (in seconds) are binned according to successive powers of ten. As shown here, all 50 problems give runtimes less than one second when CNRQ is used (likewise for CNR-1), while for CNR-LY, runtimes are at least $10^{0}$ seconds and are sometimes more than $10^{5}$.

Given the enormous difference in efficiency between algorithms based on all-solutions search and those based on counting and inference, in the remainder of the paper only the former will be considered. Some spot-checking of the latter was done in a few cases, with results resembling those in the present section. 


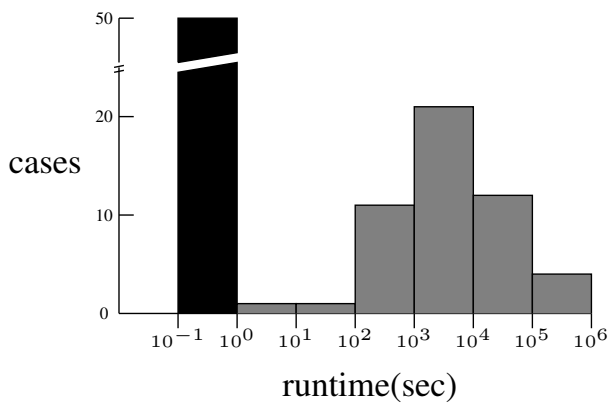

Figure 5: Frequency histogram for CNRQ (black) and CNR-LY (gray). Fifty problems in all. (Note log scale on abscissa.)

\section{Comparing CNR-1 and CNRQ}

For random geometric problems, CNR finds a large number of replaceable values, about $25 \%$ more than NIC, showing that it is effective in simplifying problems (see Table 1 ; because of space constraints only satisfiable problems are shown). For smaller problems, the reduction in subsequent search time actually compensates for the extra preprocessing time. (As a side note, it is also interesting to see how effective NIC alone was on these problems.) A similar pattern of results was found with unsatisfiable problems; in addition either NIC alone or CNR proved unsatisfiability in approximately $90 \%$ of the cases.

Table 1. Search Efficiency and Values Removed for Geometric Problems with Variable Support

\begin{tabular}{lcccc|cccc} 
& \multicolumn{4}{c}{ satisfiable-120 } & \multicolumn{4}{c}{ satisfiable-300 } \\
& rem & nodes & preproc & search & rem & nodes & preproc & search \\
\hline AC & 39 & 46,265 & 0.1 & 266.2 & 95 & 7,917 & 0.2 & 258.3 \\
NSAC & 184 & 20,906 & 8.8 & 89.8 & 492 & 2,236 & 300.3 & 81.2 \\
NIC & 301 & 268 & 36.9 & 0.7 & 924 & 1,402 & 2304.4 & 45.4 \\
CNR-1 & 370 & 269 & 149.6 & 0.7 & - & - & - & - \\
CNRQ & 370 & 269 & 67.8 & 0.6 & 1148 & 1,451 & 5269.6 & 49.1 \\
\hline
\end{tabular}

Notes. Means for values removed during preprocessing (rem), search nodes, preprocessing and search times (sec).

For the 300-variable problems, only partial results were collected with CNR-1 for both satisfiable and unsatisfiable problems. Runtimes exceeded CNRQ by up to five times depending on the problem, so that times of $10^{4}$ were observed. Hence, no attempt was made to use this algorithm on the entire problem set.

For distance problems, CNR also simplified problems more effectively than the inconsistency algorithms, as indicated by the number of search nodes. But because these problems were fairly easy, the overall processing time was much greater (Table 2). For relop problems, however, some inconsistency algorithms (but not AC) were able to remove almost as many values as the replaceability algorithms. In addition, the difference in cost was greater than with the distance problems. 
Table 2. Search Efficiency and Values Removed for Distance and Relop Problems

\begin{tabular}{lcccc|cccc} 
& \multicolumn{4}{c}{ distance } & \multicolumn{4}{c}{ relop } \\
& rem & nodes & preproc & search & rem & nodes & preproc & search \\
\hline AC & 0 & 901 & 0.01 & 0.67 & 0 & 39,659 & 0.01 & 59.32 \\
NSAC & 17 & 522 & 0.15 & 0.43 & 260 & 160 & 2.63 & 0.16 \\
NIC & 19 & 522 & 0.43 & 0.42 & 269 & 189 & 27.91 & 0.15 \\
CNR-1 & 300 & 59 & 169.48 & 0.02 & 276 & 181 & 1021.57 & 0.14 \\
CNRQ & 300 & 59 & 168.04 & 0.02 & 276 & 181 & 960.60 & 0.14 \\
\hline
\end{tabular}

Notes. Measures as in Table 2.

These tests show that many problem types are amenable to simplification by removing replaceable values. They also reaffirm the point made previously [6] that given the expense involved in carrying out these procedues, methods for inferring replaceability without search will be of critical importance.

\section{Patterns of Difficulty Across the Problem Space}

While testing neighbourhood replaceability algorithms, a pattern of effort as a function of problem parameters was discovered that is remniscent of the well-known critical complexity effect for CSP search. An example is shown in Figure 6, where a curve for CNR runtime appears along with a corresponding curve for NIC. In this experiment, constraint density is the parameter that is varied. ${ }^{3}$ As with other types of local consistency algorithms [7], the curve for NIC reaches its highest point near the phase transition. In contrast, the peak of the CNR curve is well to the left of this point in the "easy" region with respect to search effort. (Mean search effort following AC in terms of nodes was 106 at 0.5 density, 930 at 0.6 , and 518 at 0.7 , using min domain/forward-degree.)

Similar results were found with distance problems. With 'geovarsat' problems the results were not clear-cut, but in this case varying constraint density also changes patterns of connectivity (i.e. with more constraints the constraint graph becomes less clumpy); hence, it isn't clear whether complexity effects like those found with other problem types should be found here as well.

To get some insight into the basis for this effect, tests were done on the same problems where various operations and other measures were counted, in particular number of neighbourhood solutions and queue additions. (Value removals were already counted.) While value deletions and queue additions did not show any clear relation to runtimes, there was a strong correlation with the number of neighbourhood solutions, as shown in Table 3.

Why are there so many neighbourhood solutions? With increasing density, neighbourhood size increases, and therefore the number of possible tuples grows as $d^{k}$, where $k$ is the number of neighbors. But at the same time the number of constraints among neighbours will also increase. Given a particular variable with neighbourhood of size $k$, we can calculate the likelihood that an additional constraint will either produce a new neighbour or increase the number of constraints within the neighbourhood subgraph composed of the variable and its neighbours. The former is $\frac{1}{n} * \frac{(n-k-1)}{n}=\frac{(n-k-1)}{n^{2}}$, the latter is $\left(\frac{k}{n}\right)^{2}$. For problems with 30 variables, and density $=0.1$ (in terms of the proportion of edges added to a spanning tree), the average degree is 5 . This, in turn, gives a probability of about 0.027 for adding a new neighbour and 0.028 for adding a constraint to the neighbourhood subgraph. For density $=0.3$, the average degree is about 10 , and the two probabilities are approximately 0.021 and 0.111 , respectively. So we can see a shift in relatively likelihood between these two densities. By setting the two expressions

\footnotetext{
${ }^{3}$ In this case density is in terms of the edges added to a spanning tree. Thus all graphs are connected. This means that 0 represents a spanning tree, while 1 as usual is a complete graph.
} 


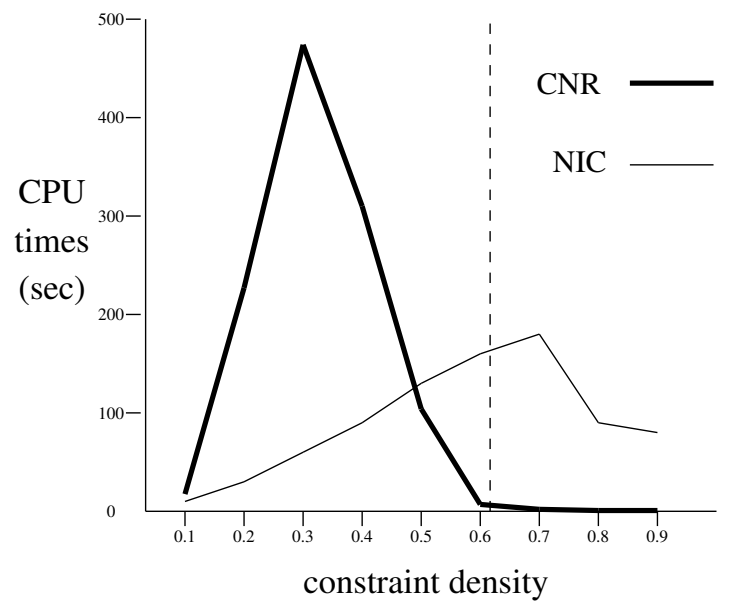

Figure 6: Runtime curves for CNRQ and NIC with relop problems of varying constraint density. Each point is the mean for 50 problems. Vertical dashed line indicates approximate transition (50:50) point from satisfiable to unsatisfiable problems.(Note. Runtimes for NIC were multiplied by 100 so its curve could be viewed on same scale as CNR.)

equal to each other, we can derive a value of $k$ equal to 5 for the crossover point for problems of this size.

Table 3. CNR Statistics for Relop Problems

\begin{tabular}{lrrrc} 
density & neigh-sols & removals & Q-addits & prove unsol \\
\hline 0.1 & 297,025 & 193 & 99 & - \\
0.2 & $3,889,129$ & 101 & 67 & - \\
0.3 & $9,564,750$ & 82 & 57 & - \\
0.4 & $6,706,596$ & 95 & 58 & - \\
0.5 & $1,809,104$ & 122 & 72 & 1 \\
0.6 & 72,054 & 152 & 70 & 17 \\
0.7 & 2,236 & 79 & 20 & 44 \\
0.8 & 5 & 14 & 1 & 50 \\
0.9 & - & - & - & 50 \\
\hline
\end{tabular}

Note. Same relop series as in Figure 6.

It might be argued that since there is no phase transition in this case, this cannot be considered a complexity peak. Although I can't yet be certain that there is a genuine asympototic complexity effect, for some classes of problems (perhaps for random problems of the RB type), this may well be the case. The implication is that complexity peaks are a more general phenomenon than phase transitions. In fact, garden path based complexity (backtrack search algorithms), support path based complexity (inconsistency algorithms), and neighbourhood solution based complexity (CNR) share a basic property. All are related to a situation where the number of possibilities increases at the same time that the likelihood that a possibility is a viable member of the desired set is decreasing.

The present difficulty peak is obviously related to one that might be found for all-solutions search (depending on the problem model and manner of search). A significant difference is that, as shown in the table, the result obtained in this case (number of deletions) does not itself increase.

Beyond its potential theoretical significance, knowledge of such a pattern of difficulty is of great practical importance in using CNR algorithms. In fact, before I was aware of this effect I had thought 
it was impossible to test certain structured problems beyond a very small number of variables, because on testing with increasing density I quickly came up against very long runtimes and had assumed that the situation would only get worse as density increased. Now we know that the limits are not quite as severe provided one stays away from the peak complexity areas.

Given that it is based on the requisite all-solutions search, it may be possible to ameliorate this effect by standard methods of checking for cycle breaking and then collecting cross-products of remaining domain values to be tested for replaceability. However, this has not yet been attempted.

\section{Inferring Replaceability: Further Discussion}

In our earlier paper we argued that for some structured problems it is possible to deduce replaceability of some of the values. In particular, for distance constraints of the form $\left|X_{i}-X_{j}\right| \otimes k$ that do not involve equalities there are several ways to avoid checking individual values. First, depending on the minimal distance, it may be possible to infer substitutability for values near the extremes, since support for extreme values will always include the support for less extreme values. (Here, we will refer to these as subset inferences.) There are also symmetry relations with respect to support for values in the lower and upper halves of the range such that if a value in one half-range can be shown to be replaceable then its complement in the other half-range is also replaceable.

However, conditions must be met to ensure that the latter maneuver is valid. In previous work we did not include a proof of this technique, and in fact there was a minor flaw in the program. In this paper we present a proof, which involves the conditions under which this strategy will give valid results.

We first consider the simplest case in which all domains contain a complete sequence of integers, i.e. there are no breaks in the sequence, and constraints are all of the form $\left|X_{i}-X_{j}\right|>k$, i.e. there is only one $k$ value.

Proposition 2. For problems based on distance constraints with the properties just given, if a value $v$ is replaceable, then the symmetric value $n-v+1$ is also replaceable provided that there is a symmetrical set of values around the midpoint of the domain.

Proof. We assume that the initial domains are complete integer sequences between 1 and $m_{i}$ for each variable $X_{i}$. (The necessary property is that for each value $v$ below the midpoint, there is a value $n-v+1$ above the midpoint.) Suppose we have found the first replaceable value $v$ for a given variable $X_{i}$. We consider any set of replacement values. Since the domain is a complete sequence, for every replacement value $w$, where $w-v=d$, there will be a corresponding domain value $w^{\prime}$ where the difference between $w^{\prime}$ and $n-v+1$ will also be $d$. In fact, if we add $d_{v-s y m m}$, the difference between $v$ and $n-v+1$, to any value $w$ we obtain the corresponding $w^{\prime}$.

Next, we consider the set of neighbourhood tuples supported by either $v$ or $n-v+1$, respectively. Note that the cardinality of these sets may differ depending on the neighbour domains. However, taking either set and some replacement set $\{w\}$ or $\left\{w^{\prime}\right\}$, the complementary set, $\left\{w_{\text {comp }}\right\}$ or $\left\{w_{\text {comp }}^{\prime}\right\}$, will be found in the domain of $X_{i}$ by virtue of the symmetry of the domain. Hence, if $v$ is replaceable it will be possible to replace the symmetrical value $n-v+1$.

Now, suppose we have replaced $k$ values in a domain, and the remaining values are symmetrical around the midpoint. Then by the same reasoning, if a value $v$ is shown to be replaceable, then $n-v+1$ can also be replaced.

This argument can be extended to constraints of the form $\left|X_{i}-X_{j}\right|<k$, or to corresponding forms where the operator is $\geq$ or $\leq$. In addition, for these problems, if a value is filtered by an inconsistency algorithm, then its symmetric complement will also be deleted. (Although AC algorithms are not able to delete any values, SAC-based reasoning (with neighbourhood SAC or higher-order SAC-based methods) is effective.) This ensures that asymmetries are not introduced by inconsistency processing. 
It is of particular interest that these arguments can be extended to problems with disjunctive constraints of the same character, which includes scheduling problems. Here, the constraints usually have the form $X_{i}+k_{1}<X_{j} \bigvee X_{j}+k_{2}<X_{i}$. The two components can be rewritten as $X_{j}-X_{i}>k_{1}$ and $X_{i}-X_{j}>k_{2}$, respectively. This, in turn, implies the constraint $\left|X_{i}-X_{j}\right|>\min \left(k_{1}, k_{2}\right)$. It follows that if for a given value $v$ all members of the replacement set satisfy the derived constraint, and the conditions specified in Proposition 9 also hold, then we can also replace the symmetrical value $n-v+1$.

In this case, however, care must be taken to ensure that asymmetries are not introduced by inconsistency processing. In the present work a very conservative procedure was used. (Whether there are more efficient procedures is an open question.) After each deletion, the symmetrical complement was the next value tested; if it was not inconsistent, then a symmetry flag for that domain was set to nil, and after that no inferences based on symmetrical values were attempted.

In earlier work with simple distance problems [6] we found that by using subset and symmetry inferences along with skipping replaceability checks for extreme values, we could reduce the time to find an irreplaceable set by three orders of magnitude, from $10^{4}$ to $10^{1}$ seconds. In the present work problems derived from the Taillard benchmarks were tested, specifically the os-taillard-4-100 set [12], which includes ten problems. Since even these problems had domains that were much too large to apply value-by-value tests, the domains were reduced to about a quarter of their original size, which is roughly from 200 to 50. (The resulting problems still had solutions.)

Despite this reduction, the basic CNRQ algorithm required an average of 30,000 seconds. A large portion of this effort was due to one problem that required 267,000 seconds to process. However, four other problems required $10^{4}$ seconds, ranging from 10 to 36 thousand.

Using either subset or symmetry inferences alone reduced the mean to 16 and 14 thousand seconds, respectively. Using these methods together along with skipping the extreme values brought a further reduction to 8 thousand seconds. Moreover, for some problems it was possible to reduce the time from $10^{4}$ to $10^{2}$ or $10^{1}$. So, while not finding the spectacular overall reduction obtained for simple distance problems, it was possible to bring about a clear reduction with these methods, which in some case was marked.

\section{Conclusions and Future Work}

This work has clarified some issues related to the important property of replaceability. In particular, we have determined what is presently the best algorithmic approach to finding a neighbourhood irreplaceable set, and we have corrected and clarified some questions in regard to inferring replaceability for constraints with certain kinds of structure.

In addition, we have discovered a new critical complexity region associated with neighbourhood replaceability algorithms and analyzed some of its features. This is of both theoretical and practical interest.

This work extends the general study of substitutability in connection with CSPs. Even more generally, it contributes to the study of higher-level properties that can be used to simplify problems.

However, we think that the real opportunities for using 'higher-level' properties such as replaceability remain to be discovered. For example, in cases where effort is made to compile CSPs into more compact forms such as MDDs, the task of establishing replaceability or other related properties may be obviated to a degree. (In fact, it should be possible to compile properties such as replaceability, so that instead of discarding such values, they are 'kept on hand' as potential substitutes.) Also, in dynamic situations where information from previous situations can be reused, it may be possible to amortize the cost of establishing high-level properties like replaceability, thus facilitating the process of finding new solutions to new problems. 
There are still many other topics that need to be considered. Most important, perhaps, is the use of replaceability in compilation techniques such as MDDs. The fact that there is no unique fixpoint raises several issues such as the existence of minimal sets of irreplaceable values and smallest minimal sets, although whether such sets can be found efficiently remains to be determined. Finally, it may be possible to find approximations to full replaceability that are almost as effective whilst being more efficient. In this connection, if the degree of the variables whose domains are tested for a property like replaceability is limited by a certain value, it may be possible to obtain a fixed-parameter tractable form of the property. Whether such variants are useful in practice is a question that must be decided in the future.

\section{References}

[1] L. Bordeaux, M. Cadoli, and T. Mancini. A unifying framework for structural properties of CSPs: Definitions, complexity, tractability. Journal of Artificial Intelligence Research, 32:607-629, 2008.

[2] F. Boussemart, F. Hemery, C. Lecoutre, and L. Sais. Boosting systematic search by weighting constraints. In Proc. Sixteenth European Conference on Artificial Intelligence-ECAI'04, pages 146-150. IOS, 2004.

[3] E. C. Freuder. Eliminating interchangeable values in constraint satisfaction problems. In Nineth National Conference on Artificial Intellgence - AAAI'91, pages 227-233, 1991.

[4] E. C. Freuder. Dispensable instantiations in constraint satisfaction problems. In Tenth International Workshop on Constraint Modelling and Reformulation - ModRef 2011, 2011.

[5] E. C. Freuder and C. D. Elfe. Neighborhood inverse consistency preprocessing. In Thirteenth National Conference on Artificial Intelligence - AAAAI'96. Vol. 1, pages 202-208. AAAI/MIT, 1996.

[6] E. C. Freuder and R. J. Wallace. Replaceability and the substitutability hierarchy for constraint satisfaction problems. In C. Lisetti C. Benzmueller and M. Theobald, editors, Third Global Conference on Artificial Intelligence - GCAI 2017, EPiC Series in Computing Vol. 50, pages 51-63. Easychair Publications, 2017.

[7] I. P. Gent, E. MacIntyre, P. Prosser, P. Shaw, and T. Walsh. The constrainedness of arc consistency. In G. Smolka, editor, Principles and Practice of Constraint Programming-CP'97. LNCS No. 1330, pages 327340. Springer, 1997.

[8] P. Jeavons, D. Cohen, and M. C. Cooper. A substitution operation for constraints. In A. Borning, editor, Principles and Practice of Constraint Programming - PPCP'94, number 874 in LNCS, pages 161-177. Springer, 1994.

[9] D. S. Johnson, C. R. Aragon, L. A. McGeoch, and C. Shevron. Optimization by simulated annealing: An experimental evaluation. part ii. graph coloring and number partitioning. Operations Research, 39:378-406, 1991.

[10] S. Karakashian, R. Woodward, B. Y. Choueiry, S. D. Prestwich, and E. C. Freuder. A partial taxonomy of substitutability \& interchangeability. In Tenth International Workshop on Symmetry in Constraint Satisfaction Problems - SymCon2010, 2010.

[11] C. Likitvivatanavong and R. H. C. Yap. Eliminating redundancy in CSPs through merging and subsumption of domain values. ACM SIGAPP Applied Computing Review, 13:20-29, 2013.

[12] E. Taillard. Benchmarks for basic scheduling problems. European Journal of Operational Research, 64:278285, 1993.

[13] R. J. Wallace. SAC and neighbourhood SAC. AI Communications, 28:345-364, 2015. 